\title{
The Prospect of Extracting Brain-Region-Specific Exosomes in the Human Bloodstream
}

\section{Evan Yang ${ }^{1}$, Andrew Neff ${ }^{2}$}

Exosomes are small vesicles, secreted by eukaryotic cells, containing molecular cargo that reflects the biochemical composition of the origin cell, including protein and RNA. Once secreted, exosomes can enter the circulatory system and be found in blood, urine, and saliva. It has been hypothesized that because exosomes contain transmembrane proteins unique to their cell of origin, specific populations of exosomes could be non-invasively extracted from the bloodstream. The protein L1CAM may serve as a marker of neuronal exosomes. However, although "neuron-derived-exosomes" could offer some specific information about in-vivo molecular neurobiology, this population of exosomes still provides a relatively noisy signal, including data on protein expression from a variety of different neuronal subpopulations. We argue that it may be possible to isolate brain-region-specific exosomes, and that data derived from these exosomes would provide a superior diagnostic tool.

\section{Background}

Like other cells, neurons secrete exosomes (Budnik, 2016). Moreover, exosome release is coupled to neuronal function: stimulating neuronal activity can increase the release of exosomes in cell culture (Faure, 2006). Supporting this finding, fully matured adult cortical neurons secrete exosomes, and the number released can be regulated by the activity of glutamate and GABA receptors (Lacehnal, 2011). Therefore, exosome activity is at least partially reflective of neuronal activity.

In the field of psychiatry, interest in personalized approaches towards treatment has been growing over the past decade (Saeedi et al., 2019). This is partially due to the varying effectiveness of treatments among individual patients. Isolating brain-region-specific exosomes could provide a step into understanding and improving patient treatment.

Already, it has been shown that the content of neuronal-exosomes, marked by the transmembrane protein L1CAM, varies based on the presence of neurodegenerative diseases, such as Alzheimer's or Parkinson's (Goetzl et al., 2016

1 Undergraduate Researcher at Emory University (UK)

${ }^{2}$ Visiting Assistant Professor of Psychology at Emory University (UK) 
and Shi, 2014). Moreover, in general, pre-clinical work has shown that neuronal exosomes contain protein and RNA that are related to mental illness (Faure, 2006). For example, SLC1A3, a glutamate transporter, can be found in neuronal exosomes (Faure, 2006) and is associated with suicidal behavior (Murphy, 2011). Another example is TUBA1A: genetic mutations of which are associated with neuroanatomical and cognitive abnormalities (Tischfield, 2011). Both TUBA1A and another neuronal exosomal protein HspA8 have altered expression levels in post-mortem tissue of suicide victims (Kekesi, 2012).

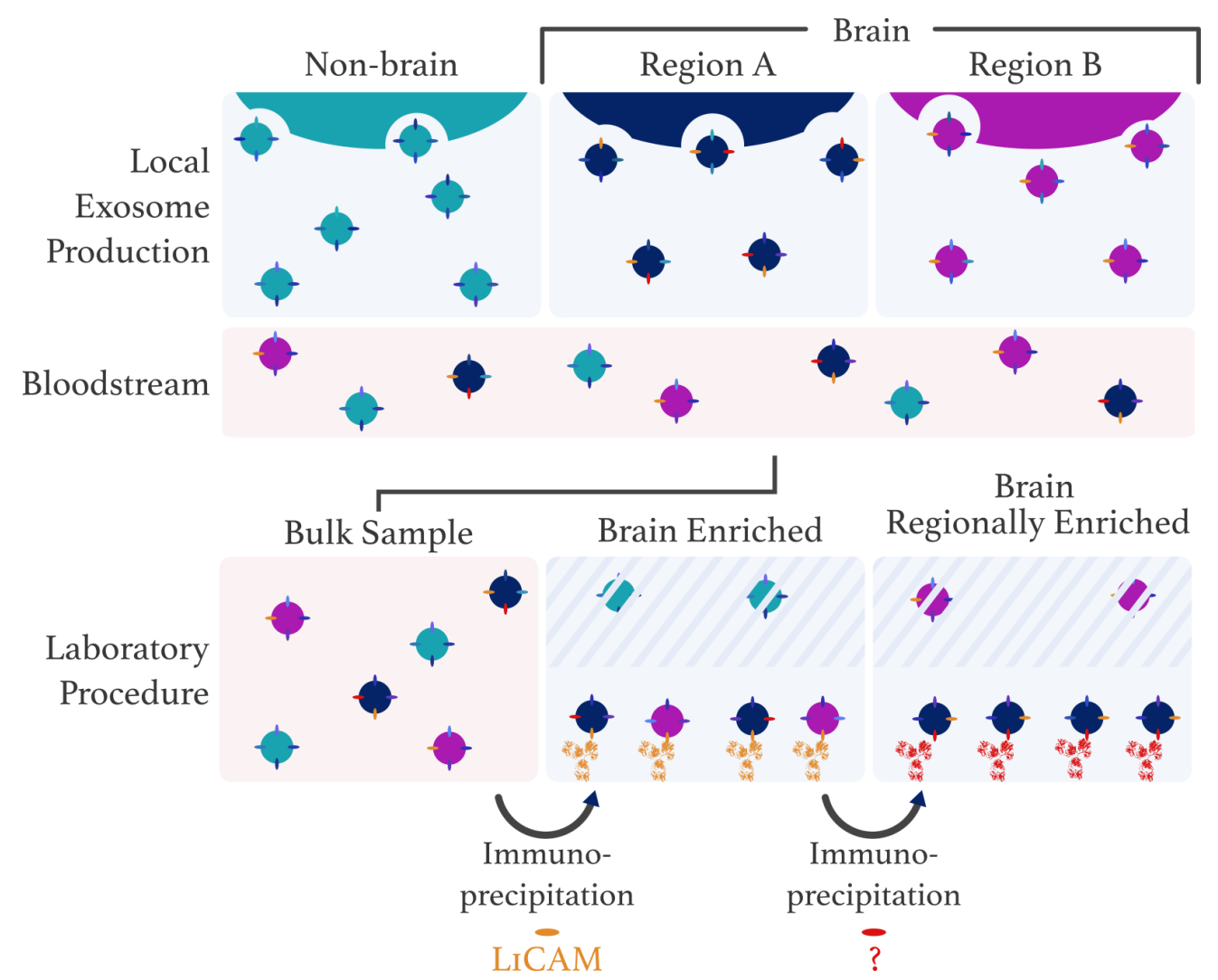

Figure 1 - serial immunoprecipitation method for isolating brain-region-specific exosomes

\section{Advancing the Specificity of Exosome Biomarker Research}

We propose a novel method of isolating brain-region-specific exosomes using a serial immunoprecipitation method.

Two simple approaches for extracting exosomes, ultracentrifugation and ultrafiltration, differentiate exosomes by size. These approaches clearly cannot be used to identify the anatomical origins of an exosome. Other techniques use immunoprecipitation in which exosomes are isolated on the basis of a specific 
transmembrane protein. Several labs have provided evidence that L1CAM is a marker of brain-derived exosomes (Goetzl et al., 2016 and Shi, 2014).

However, even existing immunoprecipitation protocols lack specificity: L1CAM is likely expressed throughout the brain (Uhlén, 2015). Centuries of research has shown that discrete brain regions exhibit different relationships with different aspects of psychology. If, hypothetically, psychiatrists were looking for a biomarker of treatment response in anxiety disorders, they may be more likely to find relevant biomarkers in the amygdala or prefrontal cortex rather than the visual cortex or cerebellum.

Abstractly, our proposal is simple: first use immunoprecipitation to extract L1CAM-exosomes from the bloodstream, then use a second round of immunoprecipitation for another protein to extract exosomes from brain-region-X.

This method relies on two assumptions that would need to be verified. The first assumption, which already has substantial support, is simply that immunoprecipitation using L1CAM isolates neuronal exosomes (see caveat 1). The second assumption is more speculative, that a second round of immunoprecipitation, using another transmembrane protein, could be used to isolate neuronal exosomes from a specific brain region or neuron-type.

\section{Caveat \#1: L1CAM Might Not Be a Good Marker of Neuronally-Derived Exosomes in Blood}

One assumption in our hypothesis is that L1CAM can be used to isolate brain-derived exosomes. Several lines of evidence do support this concept. L1CAM is a transmembrane protein found in exosomes of cultured cortical neurons (Faure, 2006). In the human bloodstream, immunoprecipitation for L1CAM (theoretically, neuronal exosomes) yields molecular markers of Parkinson's (Shi, 2014) and Alzheimer's disease (Fiandaca, 2015; Kapogiannis, 2015). The fact that exosomes reflect the neuropathology of human subjects is further evidence that L1CAM exosomes are brain-derived.

However, a recent publication has controversially called this research into question (Norman, 2021). In this experiment, researchers demonstrate that L1CAM, in human blood plasma, exists in a soluble form (unassociated with exosomes). However, these contradictory results are not fully accepted, and L1CAM continues to be used to isolate brain-derived exosomes (Hornung, 2020). Furthermore, even if L1CAM is ineffective at isolating neuronal exosomes, other proteins could theoretically do the same job.

\section{Candidates for the Second Round of Immunoprecipitation}

Assuming that either L1CAM or another protein could serve as a specific marker of "brain" or "neuronal" exosomes, a second protein would only need to provide specificity within the brain. To find a plausible second protein, we applied three criteria: 
the protein must be 1) transmembrane, 2) associated with exosomes, and 3) selectively expressed or enriched in a certain brain region.

Using a Python script, we searched two databases on exosomal protein expression, Vesiclepedia (Kalra, 2012) and Exocarta (Mathivanan, 2012), and cross-referenced those with data on regional and subcellular RNA and protein expression from the Human Protein Atlas (Uhlen, 2015) and a manual literature search. Using human post-mortem RNA and protein immunostaining data, along with exosome proteomics studies, a list of plausible protein markers for brain-region and/or cell-type specific exosomes is presented below, followed by an important caveat regarding our methodology.

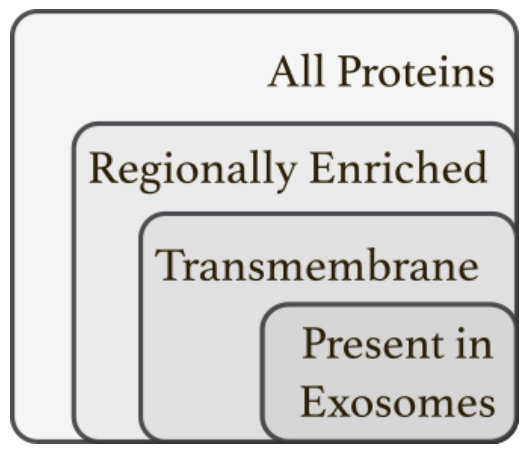

Figure 2 - criteria for identifying potential brain-region-specific marker proteins

GABRD - Cerebellum: GABRD (Gamma-aminobutyric acid type A receptor delta subunit) makes up part of one variant of the GABA-A receptor that is regionally enhanced in the cerebellum (Enoch, 2013). This protein has been identified in urine exosomes (Fraser, 2013).

GRM1- Cerebellum: GRM1 (Glutamate metabotropic receptor 1) is a g-protein coupled receptor for glutamate. It is regionally enhanced in the cerebellum, particularly in Purkinje cells (Nakao, 2019). It has been identified in serum and urine exosomes (Musante, 2012).

SLC6A3- Midbrain: SLC6A3 (solute carrier family 6 member 3 ) transports dopamine into the cell, and is regionally enhanced in the midbrain (Shimada, 1992). It has been detected in exosomes derived from T cells and in urine (Perez Hernandez, 2013 and Fraser, 2013).

SLC6A4 - Pons and Medulla / Serotonergic Neurons: SLC6A4 (solute carrier family 6 member 4 ) transports serotonin from the synaptic cleft back into the presynaptic terminal. It is only present in the pons and medulla. In a study of 5-HTT mRNA expression, it was found to be greatly expressed in the raphe nuclei (McLaughlin, 1996). In a study of platelet exosomes, SLC6A4 was identified (Pienimaeki-Roemer, 2015). Although the 5-HTT protein is likely distributed throughout the brain (Bengel, 1997), this protein could still serve as a marker of serotonergic 
neurons (and perhaps would be more valuable as such, with depression and anxiety in mind).

SLC18A2- Hypothalamus, Midbrain, Pons and Medulla: SLC18A2 (solute carrier family 18 member A2) serves as an ATP-dependent transporter of monoamines into synaptic vesicles. It is regionally enriched in those brain regions, and it has been identified in cancer exosomes (Hurwitz, 2016)

\section{Caveat \#2 - There is Not Great Data on Molecular Variation of Exosomes Across Brain Regions.}

Ideally, this project would search an atlas of exosomal protein expression throughout the brain (Created by the good people at the Allen Brain Observatory or Human Protein Atlas). Hypothetically, using post-mortem human brain tissue, exosomes could be isolated and profiled for their protein content, and this dataset would provide a list of exosomal proteins that are unique to each brain region.

In the absence of this dataset, we rely on indirect data. To confirm whether a protein is exosomal, we do not require that the protein has been found in association with "neuronal" exosomes. To our knowledge, data exists on cultured cortical neurons (Faure, 2006), stem cells (Kang, 2008), human cerebrospinal fluid (Guha, 2019), and even from the human post-mortem frontal cortex (Muraoka, 2020), but no comprehensive catalog of exosomes derived from specific brain regions exists to our knowledge. Because exosomes derived from certain brain regions may not be represented in prior studies, our only criteria is that a putative candidate for the second round of immunoprecipitation is that the protein has been found in association with exosomes derived from any cell type. Therefore, we cannot say with confidence that these proteins are found in exosomes in their respective neuronal populations.

\section{An Abstract Protocol For Experimental Confirmation}

Any further research should first seek to validate whether these proteins are found in exosomes derived from their respective brain regions. This could be accomplished with post-mortem tissue in one of two ways. Simply, each putative protein could be co-localized with exosomal markers using in-situ hybridization. Alternatively, and perhaps complimentarily, perhaps by isolating bulk-exosomes from post-mortem tissue (using ultracentrifugation) and evaluating the protein contents of the exosomal fraction.

Second, it must be confirmed that exosomes with these proteins can be found in the bloodstream. Developing the serial immunoprecipitation procedure may represent the biggest technical challenge of this project, as exosomes must be 1) captured via immunoprecipitation, 2) washed and eluted from the antibodies, and 3) further immunoprecipitated, all without damaging the structure of membrane proteins or the 
lipid structure of the exosome itself. If this procedure could be developed, the presence of exosomes can be validated in a typical way (Théry, 2018).

If exosomes with these proteins are in the blood, researchers can then further validate whether they contain molecular contents that corroborate their specific origin. Perhaps the total concentration of each RNA and protein contained within each exosomal-population can be compared to existing databases on protein and RNA expression. If at least some markers show an expression pattern similar to their cell-type of origin, that would provide further evidence for the validity of this method.

All that being said, perhaps the first step should simply be to create a comprehensive catalog of exosomal proteins throughout the brain. This catalog would allow scientists to develop more concrete hypotheses about which proteins are likely to distinguish neuronal exosomes from non-neuronal exosomes, and exosomes derived from one brain region or one cell-type from another.

\section{Conclusion}

In this paper, we propose a two-step immunoprecipitation method that could isolate exosomes from specific brain regions. The first step is to use L1CAM to isolate brain derived exosomes, the second step would be to use a second protein to isolate exosomes derived from a certain brain region or neuronal population. In this paper, we identified transmembrane proteins that could serve as plausible markers for isolating exosomes from specific brain regions. SLC6A4, as a marker of serotonergic neurons, may be particularly interesting to mental health researchers.

If confirmed, this method could provide a noninvasive diagnostic tool to create personalized treatments in psychiatry. If these findings could be corroborated, brain-region-specific neuronal exosomes could provide an unprecedented glimpse into the molecular composition of the living human brain.

\section{Gardener Comments}

\section{Ted Wade:}

"As far as I can tell, the biology of extracellular vesicles, and their smallest variety, exosomes, is a hot topic, albeit with many, many open questions. We might be a long way yet from translating this basic research into clinical applications, but the prospect of biochemical probes directly into living human brain is definitely high reward. Reliance on the L1CAM protein as a first step in enriching samples is apparently controversial, and might represent the bulk of risk in the proposed approach. The exosomes also have to survive through the second filtering step, which could mean that the entire assay technique might be quite tricky. 
One of the references (Saeedi et al) has already proposed exosome use for personalized mental health applications, so the basic idea has already surfaced. That said, relating exosome contents to mental function might need to go beyond correlations with macro brain regions or specific transmitters. Some more powerful theories now deal with the activity of multi-regional neural circuits/networks, which can now be studied in vivo in real time. To correlate such activity with exosomes would have to resolve temporal differences in the occurrence and sampling of the two measures. That seems challenging."

\section{Roger's Bacon:}

"Is it possible to modify the contents of the exosomes (with gene editing, pharmacology, or...)? I can imagine the engineering of exosomes to include proteins that are easy to isolate or have some other function (e.g. a reporter or a medicine). Exosome engineering might serve as the basis of a useful diagnostic or therapeutic tool."

\section{R. Sal Reyes:}

"This kind of noninvasive method for determining specific kinds of activity in specific brain regions could not only provide great benefits in the treatment of neurological and mental health issues, but it could also possibly provide new avenues for studying \& clarifying the functions of specific brain regions by using these methods to gather data in behavioral experiments."

\section{$\underline{\text { References }}$}

1. Bengel, D., Jöhren, O., Andrews, A. M., Heils, A., Mößner, R., Sanvitto, G. L., ... \& Murphy, D. L. (1997). Cellular localization and expression of the serotonin transporter in mouse brain. Brain research, 778(2), 338-345.

2. Budnik, V., Ruiz-Cañada, C., \& Wendler, F. (2016). Extracellular vesicles round off communication in the nervous system. Nature Reviews Neuroscience, 17(3), 160-172.

3. Enoch, M. A., Baghal, B., Yuan, Q., \& Goldman, D. (2013). A factor analysis of global GABAergic gene expression in human brain identifies specificity in response to chronic alcohol and cocaine exposure. PLoS One, 8(5), e64014.

4. Fauré, J., Lachenal, G., Court, M., Hirrlinger, J., Chatellard-Causse, C., Blot, B., ... \& Kirchhoff, F. (2006). Exosomes are released by cultured cortical neurons. Molecular and Cellular Neuroscience,31(4), 642-648.

5. Fiandaca, M. S., Kapogiannis, D., Mapstone, M., Boxer, A., Eitan, E., Schwartz, J. B., ... \& Goetzl, E. J. (2015). Identification of preclinical Alzheimer's disease by a profile of pathogenic proteins in neurally derived blood exosomes: A case-control study. Alzheimer's \& Dementia, 11(6), 600-607. 
6. Fraser, K. B., Moehle, M. S., Daher, J. P., Webber, P. J., Williams, J. Y., Stewart, C. A., ... \& West, A. B. (2013). LRRK2 secretion in exosomes is regulated by 14-3-3. Human molecular genetics, 22(24), 4988-5000.

7. Goetzl, E. J., Mustapic, M., Kapogiannis, D., Eitan, E., Lobach, I. V., Goetzl, L., ... \& Miller, B. L. (2016). Cargo proteins of plasma astrocyte-derived exosomes in Alzheimer's disease. The FASEB Journal, 30(11), 3853-3859.

8. Guha, D., Lorenz, D. R., Misra, V., Chettimada, S., Morgello, S., \& Gabuzda, D. (2019). Proteomic analysis of cerebrospinal fluid extracellular vesicles reveals synaptic injury, inflammation, and stress response markers in HIV patients with cognitive impairment. Journal of neuroinflammation, 16(1), 1-19.

9. Hornung, S., Dutta, S., \& Bitan, G. (2020). CNS-derived blood exosomes as a promising source of biomarkers: opportunities and challenges. Frontiers in molecular neuroscience, $13,38$.

10. Hurwitz, S. N., Rider, M. A., Bundy, J. L., Liu, X., Singh, R. K., \& Meckes Jr, D. G. (2016). Proteomic profiling of $\mathrm{NCl}-60$ extracellular vesicles uncovers common protein cargo and cancer type-specific biomarkers. Oncotarget, 7(52), 86999.

11. Kalra, H., Simpson, R. J., Ji, H., Aikawa, E., Altevogt, P., Askenase, P., ... \& Mathivanan, S. (2012). Vesiclepedia: a compendium for extracellular vesicles with continuous community annotation. PLoS biology, 10(12), e1001450.

12. Kang, D., Oh, S., Ahn, S. M., Lee, B. H., \& Moon, M. H. (2008). Proteomic analysis of exosomes from human neural stem cells by flow field-flow fractionation and nanoflow liquid chromatography- tandem mass spectrometry. Journal of proteome research, 7(8), 3475-3480.

13. Kapogiannis, Dimitrios, et al. "Dysfunctionally phosphorylated type 1 insulin receptor substrate in neural-derived blood exosomes of preclinical Alzheimer's disease." The FASEB Journal 29.2 (2015): 589-596.

14. Kekesi, K. A., Juhasz, G., Simor, A.., Gulyassy, P., Szegő, É. M., Hunyadi-Gulyás, É., ... \& Czurkó, A. (2012). Altered functional protein networks in the prefrontal cortex and amygdala of victims of suicide. PLoS One, 7(12), e50532.

15. Lachenal, G., Pernet-Gallay, K., Chivet, M., Hemming, F. J., Belly, A., Bodon, G., ... \& Sadoul, R. (2011). Release of exosomes from differentiated neurons and its regulation by synaptic glutamatergic activity. Molecular and Cellular Neuroscience, 46(2), 409-418.

16. Mathivanan, S., Fahner, C. J., Reid, G. E., \& Simpson, R. J. (2012). ExoCarta 2012: database of exosomal proteins, RNA and lipids. Nucleic acids research, 40(D1), D1241-D1244.

17. McLaughlin, D. P., Little, K. Y., López, J. F., \& Watson, S. J. (1996). Expression of serotonin transporter mRNA in human brainstem raphe nuclei. Neuropsychopharmacology, 15(5), 523-529.

18. Muraoka, S., DeLeo, A. M., Sethi, M. K., Yukawa-Takamatsu, K., Yang, Z., Ko, J., ... \& Ikezu, T. (2020). Proteomic and biological profiling of extracellular vesicles from Alzheimer's disease human brain tissues. Alzheimer's \& Dementia, 16(6), 896-907.

19. Murphy, T. M., Ryan, M., Foster, T., Kelly, C., McClelland, R., O'Grady, J., ... \& Brown, K. (2011). Risk and protective genetic variants in suicidal behaviour: association with 
SLC1A2, SLC1A3, 5-HTR1B \& NTRK2 polymorphisms. Behavioral and Brain Functions, 7(1), 1.

20. Musante, L., Saraswat, M., Duriez, E., Byrne, B., Ravidà, A., Domon, B., \& Holthofer, H. (2012). Biochemical and physical characterisation of urinary nanovesicles following CHAPS treatment. PloS one, 7(7), e37279.

21. Nakao, H., Kishimoto, Y., Hashimoto, K., Kitamura, K., Yamasaki, M., Nakao, K., ... \& Aiba, A. (2019). mGluR1 in cerebellar Purkinje cells is essential for the formation but not expression of associative eyeblink memory. Scientific reports, 9(1), 1-10.

22. Norman, M., Ter-Ovanesyan, D., Trieu, W., Lazarovits, R., Kowal, E. J., Lee, J. H., ... \& Walt, D. R. (2021). L1CAM is not associated with extracellular vesicles in human cerebrospinal fluid or plasma. Nature Methods, 18(6), 631-634.

23. Perez-Hernandez, Daniel, et al. "The intracellular interactome of tetraspanin-enriched microdomains reveals their function as sorting machineries toward exosomes." Journal of Biological Chemistry 288.17 (2013): 11649-11661.

24. Pienimaeki-Roemer, A., Kuhlmann, K., Böttcher, A., Konovalova, T., Black, A., Orsó, E., ... \& Schmitz, G. (2015). Lipidomic and proteomic characterization of platelet extracellular vesicle subfractions from senescent platelets. Transfusion, 55(3), 507-521.

25. Saeedi, S., Israel, S., Nagy, C., \& Turecki, G. (2019). The emerging role of exosomes in mental disorders. Translational Psychiatry, 9(1), 1-11.

26. Shi, M., Liu, C., Cook, T. J., Bullock, K. M., Zhao, Y., Ginghina, C., ... \& Zhang, J. (2014). Plasma exosomal $\alpha$-synuclein is likely CNS-derived and increased in Parkinson's disease. Acta neuropathologica, 128(5), 639-650.

27. Shimada, S., Kitayama, S., Walther, D., \& Uhl, G. (1992). Dopamine transporter mRNA: dense expression in ventral midbrain neurons. Molecular brain research, 13(4), 359-362.

28. Théry, C., Witwer, K. W., Aikawa, E., Alcaraz, M. J., Anderson, J. D., Andriantsitohaina, R., ... \& Jovanovic-Talisman, T. (2018). Minimal information for studies of extracellular vesicles 2018 (MISEV2018): a position statement of the International Society for Extracellular Vesicles and update of the MISEV2014 guidelines. Journal of extracellular vesicles, 7(1), 1535750.

29. Tischfield, M. A., Cederquist, G. Y., Gupta, M. L., \& Engle, E. C. (2011). Phenotypic spectrum of the tubulin-related disorders and functional implications of disease-causing mutations. Current opinion in genetics \& development, 21(3), 286-294.

30. Uhlén, M., Fagerberg, L., Hallström, B. M., Lindskog, C., Oksvold, P., Mardinoglu, A., ... \& Pontén, F. (2015). Tissue-based map of the human proteome. Science, 347(6220). 